\title{
The added value of virtual reality technology and force feedback for surgical training simulators
}

\author{
L. Zhang ${ }^{\mathrm{a}}$, C. Grosdemouge ${ }^{\mathrm{a}}$, V. S. Arikatla ${ }^{\mathrm{b}}$, W. Ahn ${ }^{\mathrm{b}}$, G. Sankaranarayanan ${ }^{\mathrm{b}}, \mathrm{S} . \mathrm{De}^{\mathrm{b}}$, D. Jones ${ }^{\mathrm{c}}$, \\ S. Schwaitzberg, and C.G.L. Cao ${ }^{\text {a, } *}$ \\ ${ }^{a}$ Department of Mechanical Engineering, Tufts University, 200 College Avenue, Medford, MA, USA \\ ${ }^{\mathrm{b}}$ Center for Modeling, Simulation and Imaging in Medicine, Rensselaer Polytechnic Institute, 110 8th Street, Troy, \\ NY, USA \\ ${ }^{\mathrm{c}}$ Department of Surgery, Beth Israel Deaconess Medical Center, 330 Brookline Avenue, TCC 140, Boston, MA, \\ USA \\ ${ }^{\mathrm{d} D e p a r t m e n t ~ o f ~ S u r g e r y, ~ C a m b r i d g e ~ H e a l t h ~ A l l i a n c e ~ H o s p i t a l, ~} 1493$ Cambridge Street, Cambridge, MA, USA
}

\begin{abstract}
Laparoscopic surgery requires more specialized training of the surgeons than traditional open surgery. The Virtual Basic Laparoscopic Surgical Trainer (VBLaST) is being developed as a virtual version of the Fundamentals of Laparoscopic Skills (FLS) trainer. This study assessed the current haptic and virtual reality (VR) technology of a virtual peg transfer task of the VBLaST, based on the subjective preference of surgeons and their objective task performance measures. Twenty-one surgical residents, fellows and attendings performed a peg-transfer task in the FLS and the VBLaST. Each subject performed 10 trials on each simulator. Results showed that subjects performed significantly better on the FLS than on the VBLaST. Subjects showed a significant learning effect on both simulators, but with an accelerated improvement on the VBLaST. Even so, $81 \%$ of the subjects preferred the FLS over the VBLaST for surgical training which could be attributed to the novelty of the VR technology and existing deficiencies of the haptic interface. Despite the subjective preference for the physical simulator, the performance results indicate an added value of VR and haptics in surgical training, which is expected to be demonstrated in more surgically relevant tasks such as suturing and knot-tying.
\end{abstract}

Keywords: surgical training, virtual reality (VR), virtual basic laparoscopic surgical trainer (VBLaST), fundamentals of laparoscopic skills (FLS), force feedback

\section{Introduction}

Laparoscopic surgery, as a much less traumatic operation procedure to the patient, is a more difficult surgical approach for surgeons. The degrees of freedom for manipulation are reduced from 6 in open surgery to 4 in laparoscopic surgery, which forces the surgeon's arms and wrists to end up in odd, unnatural positions. This is not only uncomfortable, but leads to muscle fatigue [2]. Haptic feedback (i.e., the sense of touch) is reduced, largely due to the indirect manipulation with laparoscopic instruments [11]. The surgeon's hand-eye coordination is severely dis- rupted in laparoscopic surgery, due to the indirect view of the operative field [3]. Other challenges in laparoscopic surgery include the fulcrum effect [1] (which results in a mirrored image between handgrip movement and the corresponding tool end-effector movement), lack of depth perception on the 2D display, and varying image magnification on the monitor. Therefore, laparoscopic surgery requires more specialized training of the surgeons in performing the basic surgical tasks.

Surgical simulators are playing an increasingly important role in surgical skills learning [9, 13], as they allow the trainees to practice their skills in a safe

*Corresponding author. E-mail: caroline.cao@tufts.edu 
environment before operating on human, and to avoid incurring the costs associated with animal models (e.g., pig models).

The Fundamentals of Laparoscopic Skills (FLS) trainer has been adopted by the Society of American Gastrointestinal and Endoscopic Surgeons (SAGES) and the American College of Surgeons (ACS) to train and certify laparoscopic surgeons in the USA [6]. The FLS is a physical box-trainer, consisting of five tasks: peg transfer, pattern cutting, ligating loop, suturing with intracorporeal knot, and suturing with extracorporeal knot. It requires a large supply of consumables (e.g., gauze, sutures, etc), is timeconsuming to administer, and provides primarily subjective skills assessment [7].

Virtual reality training has been shown to transfer effective technical skills to the operating room environment [10, 13], with the advantages of enabling objective and easily assessable scoring criteria [4].

The Virtual Basic Laparoscopic Surgical Trainer (VBLaST) is being developed as a virtual version of the FLS skill trainer box [8], with the advantages of allowing for repeated trials without the expense of consumables, haptic feedback to aid learning, and objective performance measures.

The purpose of this study was to assess the value of current VR technology used in a peg transfer task of the VBLaST, based on the subjective preference of surgeons, and their objective performance measures. It was hypothesized that subjects with more training or experience would perform better than those with less training or experience, and better than those with no training at all, when using either the FLS or the VBLaST. It was also expected that subjects' performance would be similar on both simulators, regardless of their preference for the simulators.

\section{Methods}

\subsection{Participants}

Twenty one participants with varied experience in surgery from a teaching hospital in the Boston Area participated in this study. There were 5 PGY $1 \mathrm{~s}, 5$ PGY2s, 2 PGY3s, 3 PGY4s, 3 PGY5s, 3 surgical fellows/attendings. One of the subjects was lefthanded, and 20 subjects were right-handed.

\subsection{Apparatus}

The FLS (see Figure 1A) and the VBLaST (see Figure 1B) were used to perform a peg-transfer task. Placed in the center of the FLS box trainer is a peg board with 12 pegs and six rings. The six rings are on the left side of the peg board at the start of each trial. The VBLaST simulator consists of computational software to simulate the FLS pegs and rings, and a physical interface to connect two laparoscopic graspers to two PHANTOM Omni haptic devices (SensAble Technologies, Wilmington, MA, USA). These tools allow force feedback to be transmitted to the subjects.

A digital video capture device (AVerMedia, Milpitas, CA, USA) was used to record subjects' performance inside the task space. The video was used to extract timing and error measurements for data analysis.

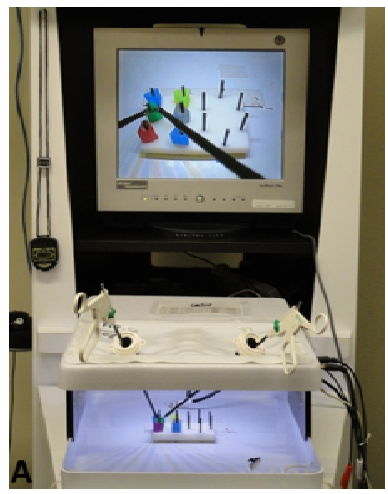

Figure 1A. FLS

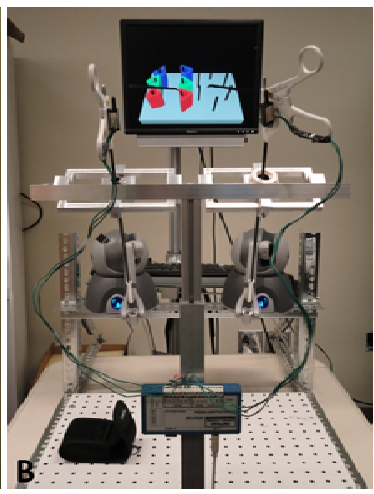

Figure 1B. VBLaST.

\subsection{Procedure and experimental design}

The peg transfer task, the first of 5 standard tasks in the FLS, was used in this study. Using two graspers (one in each hand), subjects picked up the rings (one at a time) with the non-dominant hand, transferred to the dominant hand, and placed them on the opposite side of the peg board. Once all the rings were transferred, the process was repeated to transfer the rings back to the other side of the peg board to complete one trial.

Subjects performed 10 trials of the task on each simulator. The presentation order of the simulators was counterbalanced. The experiment was a 2 (simulators $) \times 6$ (experience levels) $\times 10$ (trials) mixed design. 


\subsection{Dependent measures}

Time to task completion and error were recorded. For the FLS simulator, the dependent measures were obtained from video analysis, and a total score (ranging from a minimum of 0 to a maximum of 300) was calculated based on the established scoring metrics for the FLS task. For the VBLaST simulator, performance measures were automatically recorded by the system software and a total score was generated.

At the end of the experiment, subjects completed a questionnaire to evaluate the features of the VBLaST relative to the FLS trainer and real laparoscopic surgery, using a 5-point Likert scale.

\subsection{Data analysis}

A 3-way General Linear Model analysis (simulator, experience level, trial order) was performed, with an alpha value of 0.05. Average subjective ratings of VBLaST relative to the FLS and laparoscopic surgery were also calculated.

\section{Results}

Data analysis showed that performance on the FLS was significantly better than on the VBLaST $(\mathrm{F}(1$, $15)=55.99, \mathrm{p}<0.001$ ) (see Figure 2). Note that higher score indicates better performance.

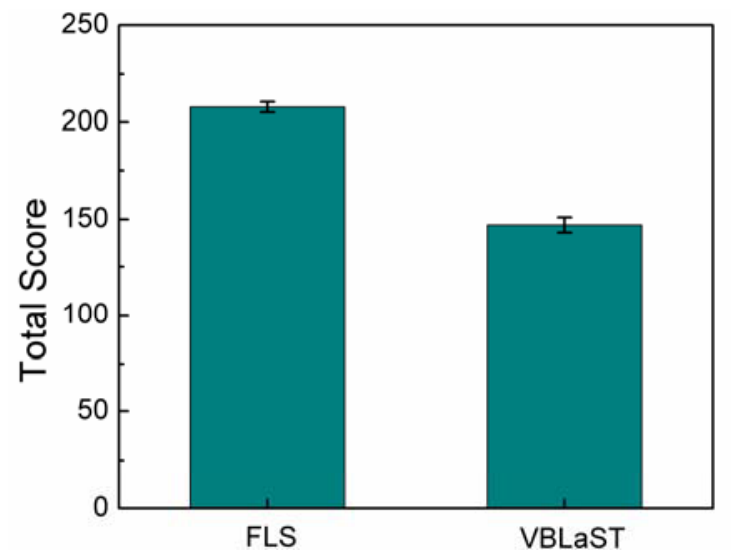

Figure 2. Effect of simulators. Error bars represent standard error.

Subjects showed a significant learning effect with trial order $(\mathrm{F}(9,135)=8.06, \mathrm{p}<0.001)$, and performance across trials could fit into a linear model for both simulators (see Figure 3).

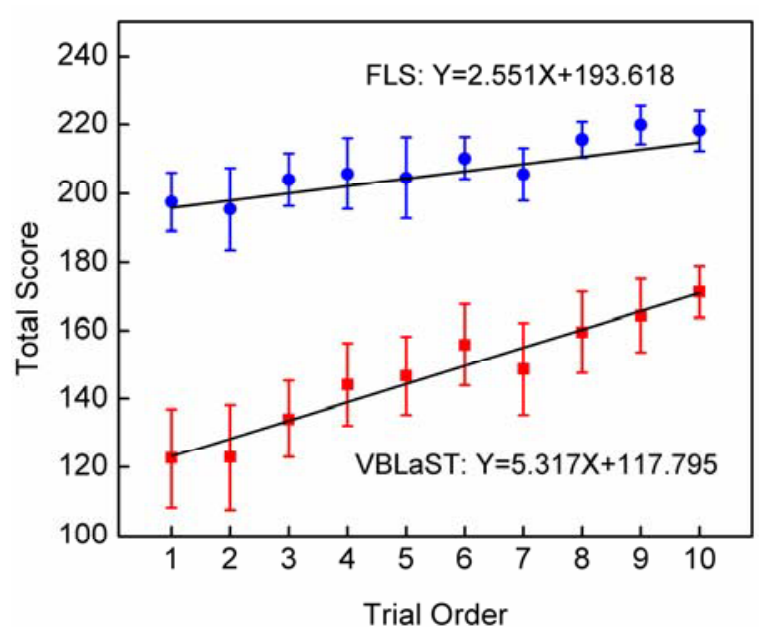

Figure 3. Increasing scores with trial number indicates a learning effect. Error bars represent standard error.

Results also showed a main effect of the experience levels $(\mathrm{F}(5,15)=4.45, \mathrm{p}=0.011)$ (see Figure 4). Post-hoc analyses showed that PGY2-5s, and fellows/attendings performed better than PGY1s who were first-year residents, on both simulators. There were no significant differences amongst the other experience levels.

No 2-way or 3-way interaction effects were found.

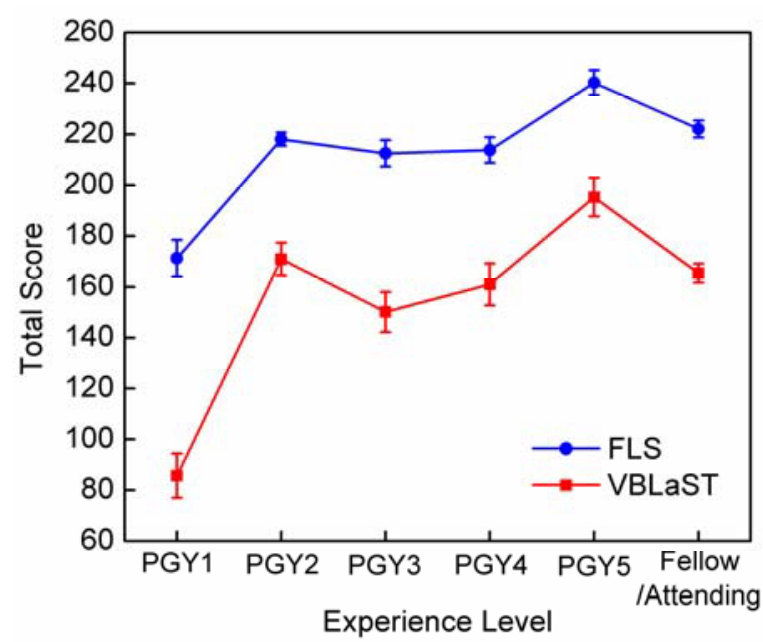

Figure 4. Effect of experience levels. Error bars represent standard error. 
Table 1.

Subjective rating of VBLaST relative to FLS and actual laparoscopic surgery on 5-point Likert scale (1=not realistic/useful; $5=$ very realistic/useful)

\begin{tabular}{lcc}
\hline & Mean Rating & SE \\
\hline 1. Realism of instrument handling compared to FLS & 2.95 & 0.23 \\
2. Realism of instrument handling compared to actual laparoscopic surgery & 2.89 & 0.28 \\
3. Overall realism compared to FLS & 3.14 & 0.20 \\
4. Quality of force feedback & 3.05 & 0.29 \\
5. Usefulness of force feedback & 3.90 & 0.30 \\
6. Usefulness for hand-eye coordination skills compared to FLS & 3.43 & 0.25 \\
7. Usefulness for ambidexterity skills compared to FLS & 3.43 & 0.26 \\
8. Overall usefulness for skills learning compared to FLS & 3.14 & 0.24 \\
9. Trustworthiness in quantifying performance & 2.86 & 0.27
\end{tabular}

$S E=$ standard error.

Subjective ratings of the VBLaST features are shown in Table 1. The score for Questions 5 (usefulness of force feedback) was the highest, with a mean score of 3.90. Questions 6 (usefulness for learning hand-eye coordination skills) and 7 (usefulness for learning ambidexterity skills) got the next highest scores, with a mean value of 3.43. Questions 1 (realism of instrument handling relative to FLS), 2 (realism of instrument handling relative to actual laparoscopic surgery) and 9 (trustworthiness in quantifying performance measures) had the lowest ratings, with a mean value of $2.95,2.89$, and 2.86 , respectively. Only 3 out of 21(14\%) subjects preferred the VBLaST over the FLS for minimally invasive surgery skills training (17 out of $21(81 \%)$ preferred FLS, and one subject did not show any preference).

\section{Discussion}

Contrary to expectation, subjects' overall performance score of the peg-transfer task on the VBLaST was significantly lower than on the FLS. It is possible that the method for evaluating performance is not adequate. The scoring system used in this comparison imposes a maximum trial time of 300 seconds, a non-arbitrary that was established based on aggregated performance data on the FLS only.
Therefore, using this scoring method allows an assessment of skill performance relative to that group of users. Thus, it may not be appropriate for application in other simulators without modification. A general performance assessment method that is not simulator-specific should be used in future comparison studies.

The lower scores on the VBLaST can be attributed to the fact that subjects have not been exposed to the novel VR technology and haptic feedback feature in a surgical simulator, and may have required more user adaptation on the VBLaST. Indeed, this adaptation effect or learning effect was observed on both simulators, with a steeper slope of the learning curve for the VBLaST (Figure 3). These results suggest that faster learning can be obtained with the VBLaST, despite the required initial adaptation to new technology. Although the data from 10 trials are not sufficient to show the intersection of these two learning curves, it is expected that training on the VBLaST can provide an accelerated performance improvement over the FLS, given an adequate amount of trials.

Subjects with higher experience levels than PGY1s performed better than the PGY1s, but no performance difference was found amongst subjects at experience levels higher than PGY1. As the most basic task in the FLS, peg-transfer may not be sufficiently difficult to demonstrate an appreciable performance im- 
provement with experience. In other words, this may be a result of a ceiling effect due to the easy task [5]. A more difficult task is expected to provide a better differentiation of experience levels based on performance.

Subjects reported insufficient realism of instrument handling of the VBLaST compared to the FLS and actual laparoscopic surgery, possibly due to the interaction between the phantom devices and the graspers. To provide force feedback to the subjects, the tool end-effectors had to be connected to the haptic device, making free motion of the end-effectors impossible.

Despite the acknowledged usefulness of force feedback and VR for surgical training in principle, the majority of subjects $(81 \%)$ indicated that they preferred to use the FLS for surgical training at the end of the experiment. Thus, the implementation of the haptic interface is an important factor in providing subjects with a sense of realism, or presence.

The potential value of haptics and VR in surgical trainers has been demonstrated by others [10, 12-14]. The results from this study, though limited, also support the incorporation of haptics in VR surgical simulation for accelerated training. However, the implementation of the haptics and VR technology must be done well, so as not to diminish the sense of simulation realism and thus disrupt the users' sense of presence. More data are needed from future studies with surgically relevant tasks, such as dissecting tissue and suturing, to examine subjective perception, and evaluate its effect on the benefits of VR simulation and haptics for skills training.

\section{Acknowledgements}

This project was supported by National Institutes of Health (NIH) Grant NIBIB R01 EB010037-01. The subjects' valuable time in this study is gratefully acknowledged.

\section{References}

[1] O. Ben-Porat, M. Shoham, and J. Meyer, Control design and task performance in endoscopic Teleoperation, Presence 9 (2000), 256-267.

[2] R. Berguer, D. L. Forkey, and W. D. Smith, Ergonomic problems associated with laparoscopic surgery, Surgical Endoscopy 13 (1999), 466-468.

[3] P. Breedveld and M. Wentink, Eye-hand coordination in laparoscopy - An overview of experiments and supporting
Aids, Minimally Invasive Therapy and Allied Technologies 10 (2001), 155-162.

[4] T. Grantcharov, J. Rosenberg, E. Pahle, and P. FenchJensen, Virtual reality computer simulation, Surgical Endoscopy 15 (2001), 242-244.

[5] L. Panait, E. Akkary, R. L. Bell, K. E. Roberts, S. J. Dudrick, and A. J. Duffy, The role of haptic feedback in laparoscopic simulation training, Journal of Surgical Research 156 (2009), 312-316.

[6] J. H. Peters, G. M. Fried, L. L. Swanstrom, N. J. Soper, L. F. Sillin, B. Schirmer, K. Hoffman, and the SAGES FLS Committee, Development and validation of a comprehensive program of education and assessment of the basic fundamentals of laparoscopic surgery, Surgery 135 (2004), 2127.

[7] E. Ritter, T. Kindelan, C. Michael, E. Pimentel, and M. Bowyer, Concurrent validity of augmented reality metrics applied to the fundamentals of laparoscopic surgery (FLS), Surgical Endoscopy 21 (2007), 1441-1445.

[8] G. Sankaranarayanan, H. Lin, V. S. Arikatla, M. Mulcare, L. Zhang, A. Derevianko, R. Lim, D. Fobert, C. Cao, S. D. Schwaitzberg, D. B. Jones, and S. De, Preliminary Face and Construct Validation Study of a Virtual Basic Laparoscopic Skill Trainer, Journal of Laparoendoscopic \& Advanced Surgical Techniques 20 (2010), 153-157.

[9] D. J. Scott, P. C. Bergen, R. V. Rege, R. Laycock, S. T. Tesfay, R. J. Valentine, D. M. Euhus, D. R. Jeyarajah, W. M. Thompson, and D. B. Jones, Laparoscopic training on bench models: Better and more cost effective than operating room experience?, J Am Coll Surg 191 (2000), 272-283.

[10]N. E. Seymour, A. G. Gallagher, S. A. Roman, M. K. O’Brien, V. K. Bansal, D. K. Andersen, and R. M. Satava, Virtual reality training improves operating room performance: Results of a randomized, double blinded study, Ann Surg 236 (2002), 458-464.

[11]H. G. Stassen, J. Dankelman, and K. A. Grimbergen, Manmachine aspects of minimally invasive surgery, Annual Reviews in Control 25 (2001), 111-122.

[12]P. Ström, L. Hedman, L. Särnå, A. Kjellin, T. Wredmark, and L. Felländer-Tsai, Early exposure to haptic feedback enhances performance in surgical simulator training: a prospective randomized crossover study in surgical residents, Surgical Endoscopy 20 (2006), 1383-1388.

[13]L. M. Sutherland, P. F. Middleton, A. Anthony, J. Hamdorf, P. Cregan, D. Scott, and G. J. Maddern, Surgical Simulation: A systematic review, Ann Surg 243 (2006), 291-300.

[14]M. Zhou, S. Tse, A. Derevianko, D. B. Jones, S. D. Schwaitzberg, and C. G. L. Cao, The Effect of Haptic Feedback on Laparoscopic Suturing and Knot-tying: A Learning Curve Study, Human Factors and Ergonomics Society Annual Meeting Proceedings 52 (2008), 880-884. 